\title{
Top Quark Properties from ATLAS
}

\author{
Christopher Young ${ }^{a, *}$, on behalf of the ATLAS Collaboration \\ ${ }^{a} C E R N$, \\ Esplanade des Particules 1, 1211 Geneva, SWITZERLAND \\ E-mail: christopher.young@cern.ch
}

Results of analyses of top quark properties measured at the LHC are presented on behalf of the ATLAS Collaboration. Particular attention is given to three recent results. In an analysis of the charge asymmetry in $t \bar{t}$ production evidence of a non-zero asymmetry is seen for the first time. Secondly, the measurement of $t \bar{t}$ spin correlations is discussed. Finally, using $t \bar{t}$ events, lepton flavour universality is tested through a measurement of the ratio of the rates of decay of $W$-bosons to $\tau$-leptons and muons, $R(\tau / \mu)=B\left(W \rightarrow \tau \nu_{\tau}\right) / B\left(W \rightarrow \mu v_{\mu}\right)$.

The Eighth Annual Conference on Large Hadron Collider Physics-LHCP2020

25-30 May, 2020

online

${ }^{*}$ Speaker 


\section{Introduction}

The top quark is the heaviest particle in the Standard Model (SM) of particle physics and measuring its properties is a key part of the Large Hadron Collider (LHC) physics program. The top-quark-pair production cross-section at the LHC is very large, $\sim 830 \mathrm{pb}^{-1}$ at $13 \mathrm{TeV}$, and this has resulted in over 100 million top-quark-pair events being produced within the ATLAS detector during Run 2. This gives a fantastic dataset with which to study the top quark.

The ATLAS Collaboration has produced a large number of results using top quark events. These include studying the production mechanism, the fundamental properties of the particle such as its mass and width, and the properties of the decay of the top quark including searches for flavour changing neutral currents. Additionally, recently, ATLAS has used top quark events to study the properties of the $W$-boson which is produced in the decay. In particular lepton flavour universality is tested by measuring the ratio of branching fractions into muons and $\tau$-leptons; $R(\tau / \mu)=B\left(W \rightarrow \tau v_{\tau}\right) / B\left(W \rightarrow \mu v_{\mu}\right)$.

These proceedings cover three recent results at the ATLAS experiment [1], the charge asymmetry in top-quark-pair production [2], the spin correlations between the top quarks [3], and the test of lepton universality in the decay of the $W$-boson [4].

\section{Charge Asymmetry}

At leading order top-quark-pair production is charge symmetric; the probability of the more forward quark being a top or anti-top quark is equal. At higher orders electro-weak effects and interference in $q g$ and $q \bar{q}$ processes result in a small predicted asymmetry where the top quark is slightly more likely to be at higher absolute rapidity [5]. Semi-leptonic $t \bar{t}$ events are selected and are divided into a resolved category which uses a boosted decision tree to reconstruct the system, and a boosted selection which requires a large radius jet which is tagged as being a hadronic top quark using jet sub-structure techniques.

Unfolded results are presented both inclusively and as a function of the mass of the $t \bar{t}$ system and the longitudinal boost. The asymmetry variable $A_{C}$ is defined by;

$$
A_{C}=\frac{N(\Delta|y|>0)-N(\Delta|y|<0)}{N(\Delta|y|>0)+N(\Delta|y|<0)} \quad ; \quad \Delta|y|=\left|y_{t}\right|-\left|y_{\bar{t}}\right| .
$$

Inclusively $A_{C}$ is measured to be

$$
A_{C}=0.0060 \pm 0.0015[ \pm 0.0011 \text { (stat.) } \pm 0.0009 \text { (syst.) } \pm 0.0005 \text { (MC stat.) } \pm 0.0001 \text { (bias.) }]
$$

which is $4 \sigma$ separated from zero giving first evidence of non-zero charge asymmetry in $t \bar{t}$ at the LHC. This is in good agreement with the next-to-next-to-leading order (NNLO) calculation of $A_{C}=0.0064 \pm 0.0006$. The differential distributions also show good agreement with the SM predictions and these are used to set limits on effective field theory extensions of the SM.

\section{Top Quark Spin Correlations}

The SM predicts that top quark pairs should be produced without polarization but with some correlation between their spin states. As the top quark lifetime is much shorter than the spin 

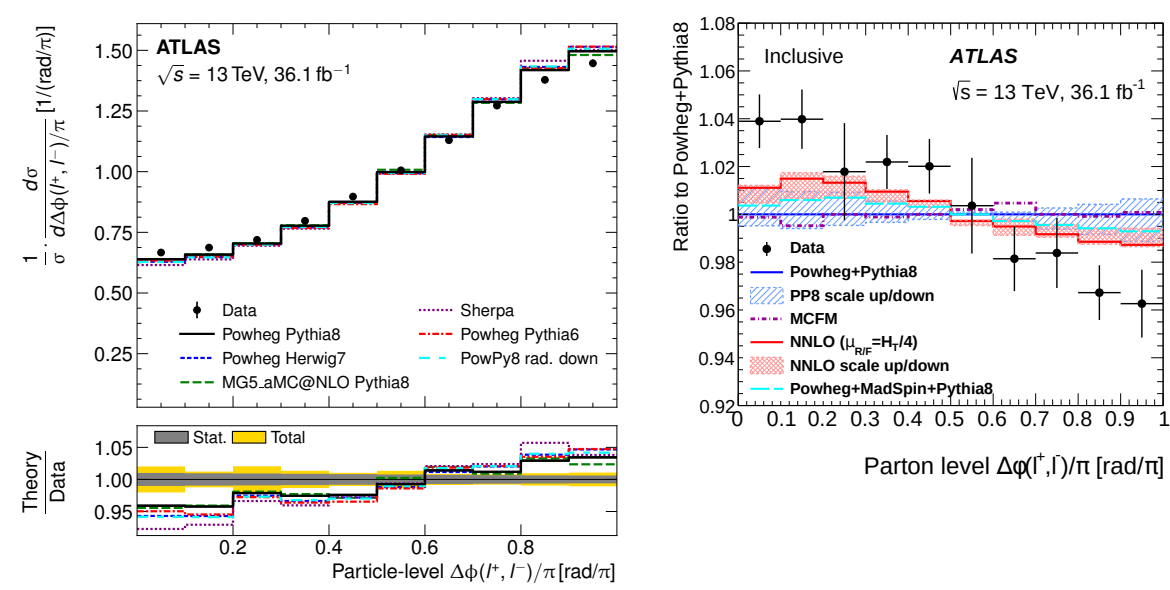

Figure 1: The normalized fiducial particle-level differential cross-section in $\Delta \phi$ compared to predictions from POWHEG, MadGraph5_aMC@NLO and SHERPA (left) and a comparison of the ratio of the unfolded $\Delta \phi$ distribution to POWHEG with additional NLO generators and NNLO fixed-order predictions (right). Figures from Ref. [3].

decorrelation time the spin information is directly transferred to the decay products. Di-lepton $t \bar{t}$ events are selected and the differential distribution of the angles between the leptons, $|\Delta \phi|$ and $|\Delta \eta|$ are studied. The data display slightly higher spin correlations than the NLO predictions but NNLO fixed-order predictions reduce this discrepancy as shown in Figure 1. Additionally the unfolded results from the ATLAS and CMS experiments are shown to be in good agreement [6].

\section{Lepton Flavour Universality}

It is a fundamental axiom of the SM that the couplings of the electroweak gauge bosons $(W, Z)$ to charged leptons are independent of the mass of the leptons. This fundamental assumption is referred to as lepton-flavour universality. This assumption is tested by measuring the ratio of the fractions of $W$-boson decays to $\tau$-leptons and muons, $R(\tau / \mu)=B\left(W \rightarrow \tau v_{\tau}\right) / B\left(W \rightarrow \mu v_{\mu}\right)$. Previously this ratio has been measured at LEP and the combination of the four experiments resulted in a measurement of $R(\tau / \mu)=1.070 \pm 0.026$ [7]. This deviates from the SM expectation of unity by $2.7 \sigma$, motivating a precise measurement of this ratio at the LHC.

Di-lepton top-quark-pair events are used to measure this ratio. One lepton, electron or muon, is used to tag the event, while the other probe lepton, which is required to be a muon, is used to measure $R(\tau / \mu)$. Muons that originate from $\tau$-lepton decays, $W \rightarrow \tau v_{\tau} \rightarrow \mu v_{\mu} v_{\tau} v_{\tau}$, are distinguished from prompt muons, $W \rightarrow \mu v_{\mu}$, through their softer $p_{\mathrm{T}}$ spectrum and the displacement of the decay through the track transverse impact parameter, $\left|d_{0}^{\mu}\right|$. The transverse impact parameter distribution in simulation is corrected using a sample of prompt muons from the process $Z \rightarrow \mu \mu$.

The normalization of the $Z \rightarrow \mu \mu$ background is derived from fitting the di-lepton mass spectrum, and the normalization of the background where the muons originate from hadron decays is taken from a selection requiring that the leptons are of the same charge. A fit is then performed to extract $R(\tau / \mu)$ in 48 bins; three bins in $p_{\mathrm{T}}$, eight bins in $\left|d_{0}^{\mu}\right|$, and two channels $(e-\mu$ and $\mu-\mu)$. 


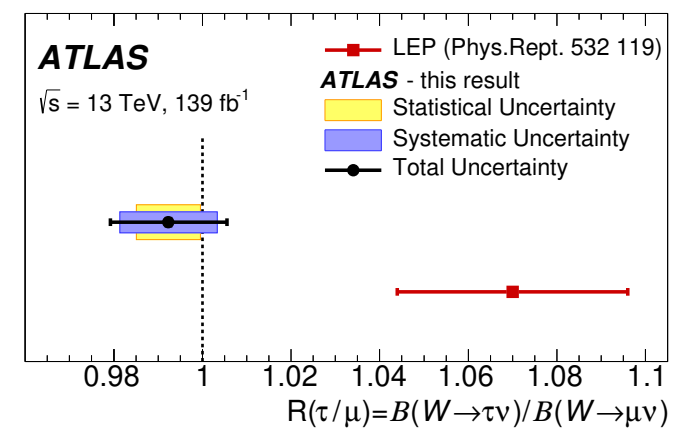

Figure 2: The measurement of $R(\tau / \mu)$ is shown (black circular marker) and compared with the previous LEP result (red square marker). The statistical and systematic errors are shown separately and also the total error of the measurement. The vertical dashed line indicates the SM prediction of lepton-flavour universality, with equal $W$-boson branching ratios to different lepton flavours. Figure from Ref. [4].

The value extracted is

$$
R(\tau / \mu)=0.992 \pm 0.013[ \pm 0.007(\text { stat }) \pm 0.011(\text { syst })]
$$

which is the most precise measurement to date. The result is shown in Figure 2 and compared with the combination of LEP measurements. The present result agrees with the SM expectation of equal couplings for different lepton flavours and the hypothesis of lepton flavour universality, and differs from the previous LEP measurement.

\section{References}

[1] ATLAS Collaboration, The ATLAS Experiment at the CERN Large Hadron Collider, JINST 3 (2008) S08003.

[2] ATLAS Collaboration, Inclusive and differential measurement of the charge asymmetry in $t \bar{t}$ events at $13 \mathrm{TeV}$ with the ATLAS detector, ATLAS-CONF-2019-026, https://cds.cern.ch/record/2682109.

[3] ATLAS Collaboration, Measurements of top-quark pair spin correlations in the e $\mu$ channel at $\sqrt{s}=13 \mathrm{TeV}$ using pp collisions in the ATLAS detector, Eur. Phys. J. C 80 (2020) 754, arXiv:1903.07570.

[4] ATLAS Collaboration, Test of the universality of $\tau$ and $\mu$ lepton couplings in $W$-boson decays from $t \bar{t}$ events with the ATLAS detector, Submitted to Nature Physics, arXiv:2007.14040.

[5] Czakon, M. et al., Top-quark charge asymmetry at the LHC and Tevatron through NNLO QCD and NLO EW, Phys. Rev. D 98 (2018) 014003, arXiv:1711.03945.

[6] ATLAS Collaboration, Spin Correlation Summary Figures from the LHCTopWG, ATL-PHYSPUB-2020-016, https://cds.cern.ch/record/2720619.

[7] ALEPH, DELPHI, L3, OPAL, LEP Electroweak Collaborations, Electroweak Measurements in Electron-Positron Collisions at W-Boson-Pair Energies at LEP, Phys. Rept. 532 (2013) 119, arXiv:1302.3415. 